\title{
Information and Life: Towards a Biological Understanding of Informational Phenomena
}

\author{
Pedro C. Marijuán
}

Fundación CIRCE. CPS, Universidad de Zaragoza. Zaragoza 50018 (Spain). marijuan@unizar.es

\begin{abstract}
This work explores a new understanding of informational phenomena based on the molecular organization of life. One of the central ideas is that the interrelationship among the recently framed fields of genomics, proteomics, and signaling science (crucial elements of the bioinformatic whole enterprise) may provide fundamental aspects of a new information synthesis. Thus, the new knowledge gained on the functionality and "existential flow" of the phenotypic molecular elements (basically the production and degradation of constituent enzymes and proteins-the transient proteome of the cell), which is intimately coupled with the intrinsic dynamics of the "DNA world" and with the communicational events stemming from the cell environment, could represent a microcosm for the whole in-formation phenomena. The variant in-formation spelling emphasizes that the cellular coupling among constitutive (proteomic), generative (genomic), and communicational (signaling) information genera produces a differentiated mode of existence, the living state, which is always in the making, perpetually in formation. The informability of the living supports the emergence of a completely new realm of 'cognitive' autonomous causality —and implies, in other regards, the emergence of meaning and of agency, and the foundation upon which far more complex, organismic,
\end{abstract}

neuronal, and social events have been evolutionarily deployed. There follows a fundamental break with respect to the mechanistic chains of causality (and explanation) afforded by the reductionist vision. There is also, in this biological approach to informational phenomena, a compelling need for the development of a new communication theory of nonconservative nature. New logical principles are discussed which could guide biological systems in their inner choices between information 'factories' and information 'garbage camps'. Finally, this bottom-up approach to the nature of information, molecularbiologically grounded as it is, does not militate against the topdown strategies. Conversely, it aims at a complementarity with germane conceptualizations that are currently being addressed in theoretical science, philosophy, neurosciences, and in social and technological disciplines.

Keywords: Information science, Reductionism, In formation, Information genera, Production-degradation, Symmetry, Knowledge, Agency, Partitional canon

\section{Introduction: The Prevailing Views on Information}

It is an irony of our times. Ushered in plain information era (proclaiming the 'information society') we still lack a scientific understanding of the i-term. One type of reasons, as we will analyze it here, is internal to the sciences, related to the excessive influence of too narrow disciplinary conceptions. Another type of reasons relates to the dominant position of information technologies in contemporary societies: once these technologies have become the most important single category of investment and are close to hold the leading position in the accumulated total of world economy, the 'tunnel vision' around information taken as a synonym of digitalization has been imparable (Zuboff, 1995; Hobar \& Schiffman, 1998; Fleissner \& Hofkirchner, 1998). The collective hype promoted by techno-utopians, i-companies, politicians, bankers, developers, etc., has stranded societies -and scientific communities at large- into another "emperor's new 
cloth" story. Why should theoreticians of the proclaimed 'information society' take care about misunderstandings and paradoxes of the i-term within the sciences (Castells, 2000)?

To say the least, an analysis of the prevailing views on information within the different knowledge environments of contemporary societies would be intellectually stimulating and quite opportune. But the social dimension of the information problem is out of the scope of this brief paper. Rather, we are going to restrict ourselves to a new conceptualization of information phenomena that is mainly based on the panorama of cellular dynamics as shown by the contemporary bioinformatic enterprise -beyond the rather naive information metaphors of classical molecular biology. In order to do that, we will discuss before, though very briefly, the current mosaic of approaches to information within the natural sciences, and will comment about the undisputed hegemony of reductionist views within that mosaic; then we will gradually introduce the conceptual suggestions related to those new bioinformatic fields.

From it to bit. With this minimalist sentence we may encapsulate the basic approaches to information in physical sciences, communication engineering, and logic and computer sciences. In fact, these are the main scientific avenues that have classically dissected the i-concept within the 'hard' sciences -or as we will argue, insufficiently dissected it. Historically, the thermodynamic approach goes first (Maxwell, Boltzmann, Schrödinger), emphasizing physical dynamics and statistical measurement of collective patterns: entropy, order, and a physical form of "state" information are the outcome. Then, communication engineering (Shannon, Wiener), that provides the famous formula for measuring the information entropy in sources and transmission channels; notwithstanding much historical confusion, this view of information is largely congruent with the former one under the guise of 'physical information theory' (and seemingly it has some more generality -Ebeling and Frömmel, 1998). Later on, two relatively independent conglomerates may be distinguished: the algorithmic-computational approach (von Neumann, Turing, Kolmogorov, Chaitin) and the logico-linguistic-semantic approach (Carnap, Bar-Hillel, Nauta, Dreske), respectively they have attempted the extension of information to dynamic laws and to symbolic realms (extracting or distinguishing forms of information into the laws of nature and the laws of thought).

Together, those approaches represent the historical grand goal of reductionism: algorithmically capturing the patterns of the material and of the symbolic, including their laws of evolution, and putting all of them into the common footing of binary strings. Thus, scientific explanation would legitimately advance, as above said, from it to bit. Or perhaps, as some physicists have proposed, the explanatory path must be laid the other way around: from bit to it (Zurek, 1990), with the world directly understandood as a gigantic computer. This would the new kind of science proposed by some parties (Wolfram, 2002), caught under very similar premises. A germane cosmological interpretation was already proposed years ago, stating that, through human agency, matter is evolving towards the greatest complexity, towards larger and deeper contents in bits, ultimately envisioning the mythical Omega point of pure information (Stonier, 1990; Abraham et al., 1995).

Those schemes, having understood information under the dominant computational form, factually grant the conceptual extension of the mechanical vision of matter and energy up to any information-related realm. Ultimately, the vision of states of matter evolving under forces impressed by the laws of nature, which is common to the three mechanics (classical, statistical, and quantum), is theoretically congruent with the timed flow of states of the algorithmic-computational approach (Rosen, 1991). Philosophically, reductionism becomes the common meta-scientific strategy, the grand vision unifying the different disciplinary strands. Reductionism directly emanates from the atomistic patterns of thought prevalent in numerous disciplines -from the widespread influence of what we may call the particulate complex of thought.

Putting aside the theoretical inconsistencies of reductionism (insurmountable ones in different fields of physics, conspicuously in cosmology, and also in mathematics themselves, or whenever the semantics is seriously considered beyond mere syntax or very shallow pseudo-semantic contexts), one of its biggest paradoxes occurs in biology, There, the multidisciplinary scheme of 'fundamental' reductive analysis has barely produced any fertile vision of the integration of information processes that characterize the 
physiology and development of biological organisms. Even at the basic molecular level, the unbalances and blind spots of reductionism have negatively oriented entire fields of biomolecular research -e.g., protein degradation, apoptosis, development, integrative physiology. The need for new guiding scientific visions beyond the unidirectional explanatory scheme of reductionism is particularly felt in the recently framed bioinformatic fields, literally struggling to make sense of an avanche of biomolecular data. The current discussions on 'systems biology', 'computational biology,' and 'functional modules' (Kitano, 2001; Hood, 2003) become experimentalists' quests to approach the processes, organization, and causality of biological systems under a new holistic orientation complementing the reductionist strategy.

To put it in a more philosophical vein: How can the reductionist bottom-up, molecularly grounded approaches to causality be consistently intertwined with the top-down directions of wholistic causality as enacted by living beings? Perhaps throughout a new understanding of informational phenomena. That is the basic theme we are going to explore in the rest of the paper. Understanding the 'invisible hand' of information should not rest exclusively on a vast catalog of computational processes and models addressing the physico-molecular occurrences, but also on making an integrated sense of the differentiated way of existence that the living system enacts. This implies a new interpretation of very basic themes of the contemporary biology of the cell.

As we are going to argue, the cellular condition of adaptively combining production and degradation processes leads to an 'evanescent permanence' of the component biomolecular structures which is genetically grounded and performed in continuous communication with the environment. The underlying interplay of the constitutive, generative and communicational forms of information in the living cell, we will argue, becomes a central organizing phenomenon that transpires into the interrelationship between the corresponding fields of bioinformatics. In the rest of this paper, apart from developing these ideas and adding further elements to a conceptual cluster centered around biological information, a mathematical hypothesis on the characteristic signature of information processes in the living cell will also be explored. It revolves on the possibility that an entropy-like statistics appears as a natural canon to which cells and other informational entities tend to abide along their adaptive interaction games.

\section{Cellular Organization}

In what extent does the living conform to the prevailing reductionist views on information? Echoing the late historian L. Kay (1993), the bioinformatic enterprise represents the historical triumph of the computerreductionist approach to life. The information metaphor enshrined by the founding fathers of molecular biology -expression, transcription, code, translation, messengers, transference, signaling, and so on- has finally given way to the projection of the biological stuff within the disembodied binary bit. As someone has recently put, 'the living is digital' (Hood \& Galas, 2003).

However, even a cursory glance on the current goals and progresses in the postgenomic biology shows vast realms of genuine 'biosemiotic' interrelationships among the variegated elements and processes integrated into the new fields of genomics, proteomics, signaling science, structural biology, transcriptomics, metabolomics, etc. Even the most simple metabolic question becomes like searching among partially overlapping entries distributed among endless dictionaries (Overbeek, 1999; Marijuán, 2002). Rather than reduction or reductionism, it is integration or integrationism the catchword epitomizing the daunting tasks ahead for bioinformatic subdisciplines.

Another caveat, of more fundamental nature, about the biological relation with reductionism is that, at the time being, no amount of syntactic structures or computing power may bridge the great gaps that still separate life processes from truly formal procedures: the molecular structure(s) of water, the protein folding problem, and the non-picturable function of the enzyme (Conrad, 1989, 1996; Marijuán, 2002, 2003). Besides, the tactilizing processing performed by numerous complexes of enzymes and proteins, the protein degradation phenomena, and the massive superposition of information gradients that occur within the water milieu constitute, in their own, non-reducible phenomena directly connected with the 
previous fundamental gaps. The biological order of processes is precisely founded upon 'emergences' stemming out from all these non-tractable problems.

Advancing the integration path does not mean the simple contrary of analysis, as life's information processes cannot be reduced to an endless accumulation of fractionable molecular mechanisms. In this sense, coherently approaching the dynamics of adaptability in informational terms may be one of the most interesting 'integrationist' tasks to initially envision, for adaptation is itself a crucial concept in theoretical biology, interrelating the functionality of populations of molecules and their environment. Two phenomenological aspects of cellular adaptability will be addressed then: the functional coupling among the different information genera, and the central (but almost unnoticed) role of degradation phenomena in the living cell.

\subsection{The Cellular Coupling of the Three Information Genera}

Let us start by restating the intriguing parallel that can be drawn between three of the bioinformatic fields -perhaps the most crucial ones: genomics, proteomics, and signaling science- and the three information genera established during the FIS discussions (see, Marijuán, 1998-fis web pages archive: http://fis.iguw.tuwien.ac.at/mailings/): constitutive, generative and communicational. These three genera were inspired by the very different types of information processes that regularly take place within living cells. Arguably, some parties could contemplate that these three genera have as a forerunner the semiotic work of Charles S. Peirce (1931).

In point of fact, the coupling among the three fields/genera illustrates a very basic picture of cellular adaptability. A constitution of enzymes and proteins (a proteome) interacts with its environment and detects and processes ad hoc signals (signaling science) which converge on a specific activation pattern of gene families (genomics), subsequently changing the present population of enzymes and proteins (changing the proteome), and then the new signals to pick up, etc. Cellular adaptabilitiy, echoing Conrad's adaptability theory (Kirby, 2002), would hinge on the functional relationship between those three omes (proteome, genome, signalome).

We could tentatively apply Conrad's entropy functions to the ongoing molecular adaptability of a cell in the organismic context as follows (Marijuán, 2003):

Range of adaptive possibilities $=$ Prot $\times$ Gen $/$ Sign

$$
\text { Adaptability = Entropy [Prot]+ Entropy [Gen]- Entropy [Sign] }
$$

It makes sense stating that, at any moment, the structural, generative and communicational aspects of the cell are caught in a web of mutual interactions in order to distribute adaptively, consistently in a topdown fashion, the functional activities of the cellular organization among the selected population of molecules (out of the many possible ones).

The negative computational role played by the signaling system, congruent with Conrad's tradeoff, can be easily recognized in the cellular system by paying attention to the signaling system's work performed within the nucleus. Along the developmental path of every organismic cell towards specialization, the signaling activities actually collapse transcriptomic diversity in the nucleus, as most of the genome is finally caught into a heterochromatin repressed state, see Gasser (2002) for discussion and very illustrative pictures. In spite of its utter simplicity, analyzing the physiological dynamics of any particular tissue, e.g., neuronal one, in the previous adaptability terms shows intriguing results (Marijuán, 2003). 
By following this path, a neuro-molecular connection with the Weber-Fechner's law on neuronal 'sensation' as a logarithmic function of the stimulus intensity looks feasible (do we perceive but neuronal 'entropy functions'? - see Kirby, 2002; Marijuán, 2003).

\subsection{The Cellular Balance between Production and Degradation}

As a result of the coupling between the three information genera and the retinue of associated energy transformations, life is always in the making. So to speak, the cellular system is always witnessing the emergence of its selected population of adaptive molecular elements through the narrow tunnel of the ribosome. Actually, masterminding alien ribosomes (or directly capturing the product of their work) is a universal target for all forms of life, either by infection, symbiosis, predation, multicellular cooperation, etc.

Just looking at the ribosomes, the whole cell has often been compared to a factory of mass-production, the output of which is a large collection of active elements that immediately integrate themselves into the factory workings. Theoretical reflection on this auto-production property of life, molecularly well established during the 60's, has induced biological thinkers to the creation of ad hoc new terms, such as autonomy, autopoiesis, self-production, self-transcendence, self-reference, auto-catakinesis, autogenesis, etc.

At the same time, however, life has to witness the disappearance of these active elements themselves, for instance, once they have spent their average working life or have prematurely lost their functional capabilities. As was discovered during early 90's, crossing the gates of the proteasome complex represents the terminal event for misfolded proteins, those suffering from 'wear and tear', oxidized and degraded ones, those marked by ubiquitin tags or by PEST sequences, $\mathrm{N}$-terminal rule, etc. Then, their constitutive amino acids are either recycled into the cellular pool or metabolized. A host of functional processes in unicellulars and multicellulars (including apoptosis itself) are related to this orchestrated degradation of enzymes and proteins.

Although proteasomes are big multimeric complexes (on the order of 2 Megadalton, almost half the size of a ribosome) they passed unnoticed until the 90's. Very scant theoretical reflection has been devoted to the analysis of the cellular creative destruction that necessarily accompanies the constructive mechanisms of ribosomes and dynamically balances them (Marijuán, 1996). Again, most of the aspects distinguishable in this property have a top-down nature, and they are usually performed and orchestrated from the highest levels of cellular organization.

Let us precisely build our integrated vision of biological information upon the 'enlarged adaptability' that derives from the dynamic balance between production and degradation. Let us highlight that the functional interrelationship among the three information genera (constitutive, generative, communicational) conduces, not to an unidimensional process of growth within the entity -or to a monotonous accumulation- but to an informable, adaptable, smart organization. The living entity is capable of continuously keeping itself in a state of change, ceaselessly tinkering with its biomolecular form, always keeping itself in formation, by appropriately communicating with its environment and adequately combining the creation of new structures with the trimming down of obsolete, unwanted parts.

\section{Being In-Formation}

By being continuously in a state of change, in formation, the living cell may adapt itself to a multiplicity of environments or to environments of any complexity, i. e., to the development of organismic specialized functions. The (low-energy) communicational information the living entity continuously exchanges with its environment orientates ('informs') its own structural flow, and tips the dynamic balance between its (highenergy) production-degradation activities on which its population of active elements, internally encoded, depends (Marijuán, 1996b; Barham, 1996). 
All this is possible, let us emphasize, because of the different cellular varieties of information (as said, information is at work simultaneously as communication, structure, and blueprint) and because of the balanced coupling between production and degradation phenomena. It remains unclear, however, the extent to which this type of adaptive, information-based modality of organization occurring in biological systems (unthinkable in the comparatively 'shallow' physical systems) can be taken as a paradigm of further informational existences.

Thermodynamically, being continuously in a dynamic balance of construction and degradation, in formation, waiting for the triggering signals of the environment that precipitate the adaptive form, becomes a very onerous modality of existence. But, at the same time, it conveys an extraordinary capability of adaptation. This is precisely the way living beings have managed to propagate themselves across time. Actually, the terrestrial abundance of free energies and the creativity inherent in the alterations of the generative information have made possible the exploration of almost any kind of evolutionary organization: from procaryotic cells to eucariots, multicellular organisms, conspicuously nervous systems, ecological niches, and then animal and human societies; the latter with their extraordinary diversity of social, cultural and economic structures.

Most of these amazing existentialities emerging out from the biological underground, and paradoxically included by the reductionist approach under the umbrella of their 'particulate complex', appear as evanescent -informational- entities involved in an unending dance of their constitutive structures, continuously immersed in the process of re-creating themselves according to conglomerates of tiny signals that they exchange with their environment, basically composed by other informational entities also in the making (let us think, for instance, on enterprises competing in a market).

In purity, we could propose a new spelling for the information term, literally meaning the capability of being in formation. It would refer to the paradoxical 'evanescent permanence' that is achieved by selfrecreation through signals impinging upon the maintenance of a dynamic balance between production and degradation activities. (Could the idea of 'dynamic balance' sustain, or serve as a substrate of, a set of irreducible properties? Given that different organizing elements would suffer their turnover at very different rates, the slower ones may perfectly become 'references' or irreducible 'identities' for the fast changing ones; thus, the multi-time nature of the aggregate dynamic balance regime admits considerable layers of conceptual complexity).

In the extent to which reflection on this paradoxical dynamics of the biological entities may coalesce into a unitary conceptualization, then the new spelling for the term 'information' would be a very fitting conceptual option for the establishment of a new interdisciplinary point of view.

\section{A New Conceptual Cluster around Information}

A cluster of co-defining terms would be necessary for further consolidation of this new approach to the nature of biological information, particularly if we are willing to advance towards the analysis of further informational existences beyond the immediate cellular realm (we have already mentioned economic organizations-enterprises, let us also think on nervous systems and societies at large). For clarity reasons, some of the accompanying concepts will be discussed only in the cellular context (their extension or generalization to other realms is not immediate). In all cases, the concepts discussed appear closely related to the different forms of information dynamics in the living cell.

Thinking on the constitutive or structural aspects, it is symmetry the term that has to accompany information most closely. In fact, both terms co-define in most theoretical contexts (information appears as a result of symmetry breaking, while symmetry refers to the permanence of pre-existing information). Probably, the idea of dynamic balance which characterizes the flow of most cellular structures -finally related to the biochemical equilibrium implicit in the Law of Mass Action- is formally amenable to the symmetry approach (Marijuán, 1996c, 2002). 
Then, some new considerations on entropy would be desirable (Lin, 2001), so that the specific occasions of structural information or symmetry-breaking in the cellular context might be conceptualized as entropy of mixing outgrowths or functional voids. Solving these occasions of inner 'voids' usually means needs or problems that are preferentially 'filled in' by the dedicated productive machinery of the cell (degradation is comparatively more onerous, as it implies a previous synthesis, although in the long run production and degradation processes intertwine rather harmoniously -e.g., in the mature, non-growing organism). There would also be an implicit reference for both information and symmetry (and symmetry breaking) in the cellular realm, but it would be a changing trajectory, a 'curvilinear' one: the cellular life cycle (Igamberdiev, 1997). Actually cycles are of utmost importance in the overall orchestration of any living structures, a fundamental tool in their multifarious synchronization of events both in energetic and informational grounds (Ho, 1995). Cycles become a necessary tool to conceptualize the general dynamics of 'autonomous systems.'

The generative or blueprint aspect of cellular information would demand the concept of symbolic representation (covering the crucial fact that by altering single nucleotides in the genome the cell can modify the non-picturable function of its enzymes in the proteome); the 'DNA world' turns into a 'knowledge repository' as, after the intervention of multiple rounds of natural selection, the symbolic representations become knowledge, implying at the same time an efficient command upon aspects of the environment and success in the advancement of the life cycle (Marijuán, 2002).

In order to perform their efficient action, the different pieces of knowledge have to mutually dovetail within integrated, or cohesive, functional modules or clusters. So any biological 'function' performed by the active elements of the cell appears pregnant with a dense 'networking' interrelationship as well as with a 'cognitive' or teleological referent. This effective 'delocalization' of the knowledge implicit in the biological function underlies the current discussions in bioinformatics on systems biology (Kitano, 2001; Marijuán, 2003). Further refining concepts such as the action/perception cycle may enter into the generative picture too, particularly once the bridging role of cellular signaling systems in the communication of the living structures with the environment is acknowledged.

Meaning and agency would be general consequences of coupling the self-production activities of the living with the arrival of external signals. Respectively, they would represent the structural changes motivated by the incoming signals, and the emergent capability to integrate action and perception in the fulfillment of a life cycle.

In the living cell, communication issues become closely related to the ongoing structural adaptations by the communicating cellular subjects (so, a difficult context for the disembodied Shannonian conceptualizations, that just refer to the message exchanged and not to the state of the producer or the receiver subjects). More concretely, approaching communication through cellular signaling systems puts the emission or reception of messages in a peculiar light, as any living cell is surrounded by an enormous range of possible exchanges with its environment; and the cell needs efficient communication strategies conducing to a massive destruction of useless, non-relevant information items. The abduction concept is a useful notion to refer to such active strategies in a global way.

However, the most important biological questions would relate to the system-logic behind the operation of both 'factories' and 'garbage camps' in the ongoing communicational process. Unfortunately, classical information theory is conservative in that regard (only 'redundancy' allows the pruning down of irrelevant parts of the message). Therefore, this cursory reflection on the communication aspects of cellular information leads to a wider theoretical quest about new formal approaches to the 'structurally grounded' modality of communication processes. A very preliminary reflection on cellular signaling systems and their relationship with the ongoing enzyme networks and gene expression patterns (how a consistent processing of the incoming information in each cellular context leads to an adaptive pattern of gene expression) was already explored in Marijuán (1994). 


\section{A New Theoretical Approach to the System-Logic of Cellular Communication}

It is upon the two traits above mentioned -necessity of a structure-grounded approach to cellular communication, and necessity of a non-conservative information theory- that this author and his colleagues have explored an alternative path based on the theoretical tool developed by Karl Javorsky, known as multidimensional partitions (Javorszky, 1995, 2000, 2003). Notwithstanding our complete disagreement on the biological interpretations of his partitional views, Javorsky's approach may be considered as a useful tool for analyzing cellular communication, provided that three logical principles are added, which we have dubbed as economy, parsimony, and symmetry (Marijuán \& Pastor, 1998; Marijuán \& Villarroel, 1998; Villarroel, 2002).

Of these three principles, only economy would be partially shared with the Shannonian approach. The three logical principles (equivalent to discarding redundant items, discarding differences already taken into account, and discarding any positional difference among the items) together achieve a considerable simplification upon the exchanged 'soup' of messages, so that finally a consistent skeleton of logical 'distinctions' may be obtained among the surviving parts within the overlapping elements of the message (cellularly, let us think on collections of overlapping gradients impinging upon ligand-gated receptors or upon multimodulated enzymes).

The guiding idea is that, by following the new communicational logic, one can apply 'multidimensional partitions' as formal descriptors of the messages. These new partitions are themselves built out from the combination of unidimensional partitionso (additive decompositions of numbers); e.g., in the case of 5 its unidimensional partitions are:

5 ,

4.1,

3.2 ,

3.1.1,

2.2.1,

2.1.1.1,

1.1.1.1.1

Seemingly, one could use these additive decompositions as mathematical descriptors of the emerging communication state-space, not much differently from Cartesian multidimensional coordinates (Marijuán et al., 1998). We cannot explain further details here; however, in the extent to which this new approach to cellular communication might illuminate cogently some parts of the 'bio-logical' interactions between the cell's environmental events and the subsequent patterns of gene expression, then the new conceptual core could be used to explore its application into other biological and non-biological arenas. (Cellularly, it is probable that the Law of Mass Action itself and other populational properties of biomolecules might be interpretable through partitional dynamics too.)

Further partitional hints of general nature about the coupling between structures and communication processes are introduced in the next section. There is little doubt that, in comparison with the relative robustness of the structural and generative aspects, communication processes become the 'cream' of the informational entities. Communication is the subtle space where most evolutionary (and social) inventions have been deployed, and the difficult subject where theoretical innovations have to be most actively pursued. 


\section{Mathematical Dignature of the "Invisible Hand"}

The ubiquity of power laws (Zipfz's law, Pareto's law, organismic allometries) in the most varied settings, physical, vegetal, animal, neuronal, social, economic, linguistic, and technological, is an amazing fact widely recognized. Recently, this has been interpreted mostly under the assumption of self-organized criticality emerging from various types of dynamic equations (Bak, 1996). Also, late Gordon Scarrott (1998) has pointed out recursive organization as a basic trait in the emergence of human information systems, impling the subsequent presence of hyperbolic (or power law) distributions in the most varied structural, communicational and economic contexts. Other conceptualizations related to power laws have been attempted, mostly in the biological (e.g., based on metabolism and internal transport).

The view of this author, in relation with the above partitional stuff applied to communication purposes, is that the adaptive game of changeable structures, in which are involved informational existences, actually conduces to the exploration of almost any conceivable compositional outcome, to the occupancy of any possible partitional state. In other words, variable structures trying to keep their 'balance' under the impact of communication exchanges would be globally incurring in a partitional dynamics too.

Interestingly, a cursory glance to the relative presence of any of the sub-summands in the total partitional of a number $\mathrm{N}$ shows a distribution close to the power law (eg, for $\mathrm{N}=5$, the relative presence of sub-summands $1,2,3,4$, and 5 with respect to the union of all possible partitions of 5 seen above, 5.4.1.3.2.3.1.1.2. 2.1.2.1.1.1.1.1.1.1.1 intuitively looks very close to a power law). Actually, there would appear: one sub-summand 5 , another 4 , two 3 , four 2 , and twelve 1 . See Table 1.

Our exploration of successive partitions of $N$ (Marijuán et al., 1998), up to $N=50$, has shown a large zone of regularity in the power law distribution for low and middle value of the summands, but an accompanying tail of exponential law (or exponential cut-off) for the highest summands. See Figure 1 and Table 1. For example, in the above partitions of $\mathrm{N}=5$, the summands $1,2,3$ and partially 4 would accommodate to the power law distribution, while 5 itself and partially 4 would be close to the exponential. Understandably, the exponential cut-off was introducing, in our opinion, an unwanted contamination, although full of regularity in any case (the graphic representation was showing an almost crystalline order -Marijuán et. al., 2001; García-Olivares \& Marijuán, forthcoming). See Figure 1 and Table 1.

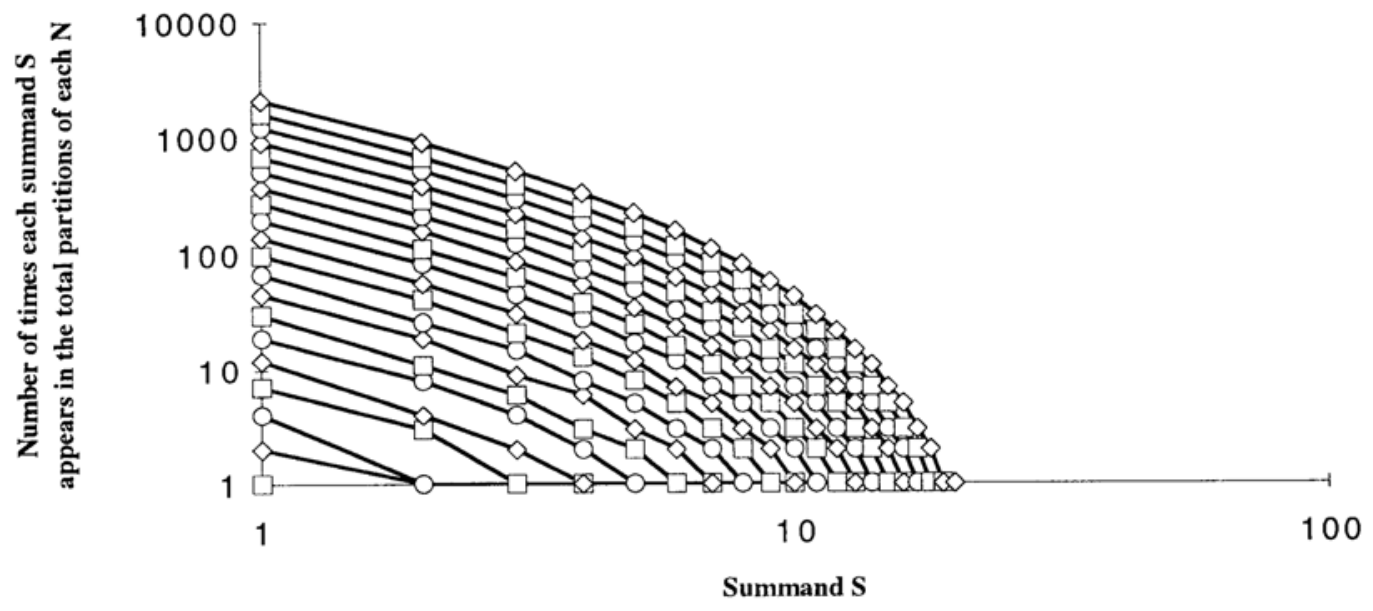

Figure 1: Power laws in partitions. For each number $\mathrm{N}$ starting from 1 until 20 there is a curve that indicates the number of times (in ordinates) that each summand $S$ appears in the total partitional of the number. The $\mathrm{x}$-axis represents the summand S. 


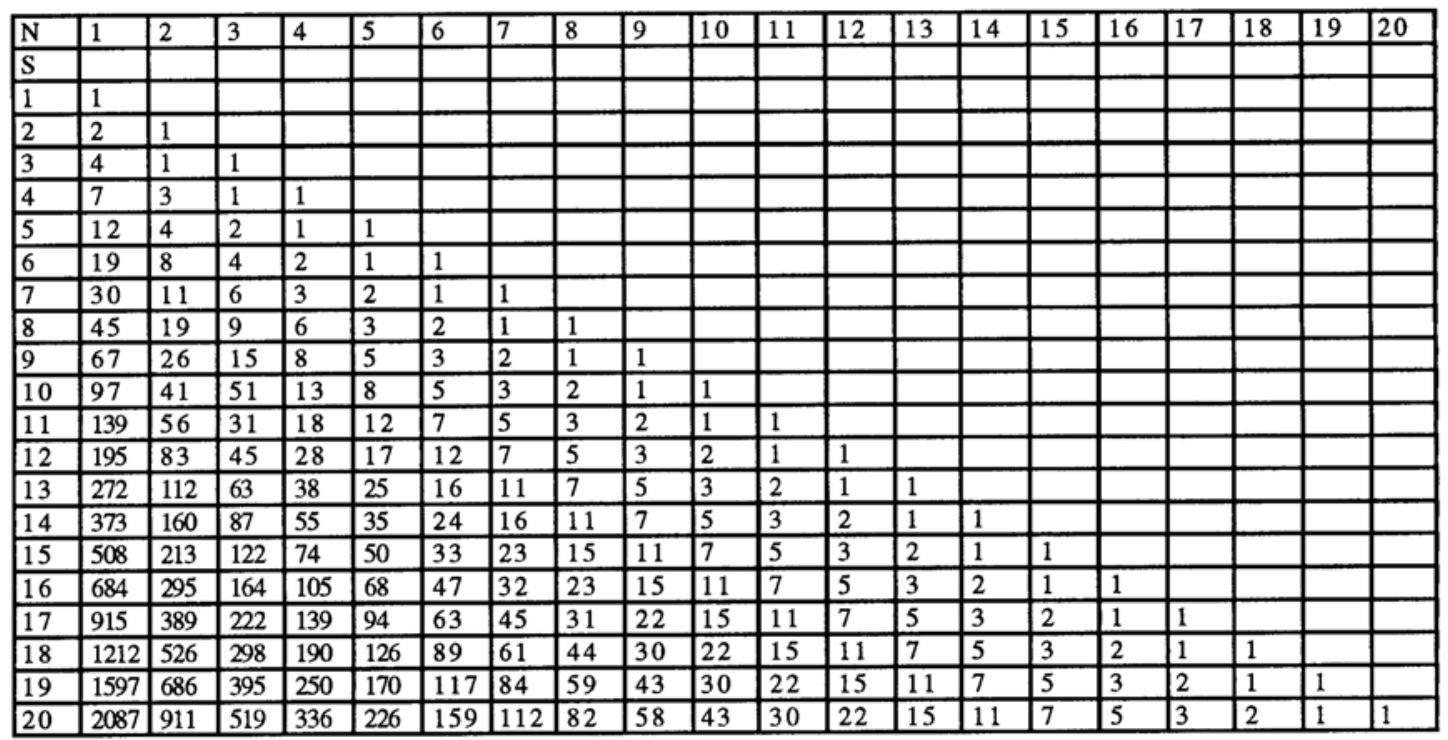

Table 1: The same information on $\mathrm{N}$ and $\mathrm{S}$ than the above curves, now put into the form of a matrix. The first column indicates values of $\mathrm{N}$ (from 1 to 20 ) and the successive columns indicate the number of times that appears each summand.

Later on, our accidental discovery that numerous cases of power law in the literature also contain an 'unwanted' exponential cut-off similar to our partitional canon (the term chosen for the distribution of the total partitional sub-summands) has suggested us the possibility of a hidden order. It is the hypothesis that 'mature' distributions of populations of informational entities might abide to a natural statistics: the partitional canon itself. Actually, this canon represents the easiest outcome of the easiest operation: a populational random walk through a landscape of successive minimal additions and substractions in the structures of the participating agents.

The suspicion (Gell-Mann, 1994) that an entropy-like distribution related to a deeper phenomenon could underly the natural universe of power laws, has been interpreted by us in a similar way to the MaxwellBoltzmann statistics to which populations of gases do abide. Populations of self-constructing agents (informational ones) endowed with enough organizational freedom tend to mature, as if pushed by an invisible hand, towards the partitional canon -a power law with an exponential cut-off. This is the hypothesis that these authors are going to check out (Marijuán et al., 1998; García-Olivares \& Marijuán, forthcoming).

Partitions, discovered long ago by mathematician Leonard Euler, nowadays are a hot topic of research. The author we have already mentioned, Karl Javorsky, has produced a number of intriguing mathematical structures along his original path of discovery in this new field (e.g., his System M series of quasiFibonacci numbers -Javorszky, 2003). Besides, Morris Villarroel (2002) has found amazing group structures that emerge after the application of the three above mentioned principles of economy, parsimony, and symmetry in a universe of two-dimensional and three-dimensional communicating messages. Perhaps it is not too farfetched thinking that a variety of natural (and mathematical) structures and constants are waiting to be rediscovered along the partitional track. We have also found that, quite probably, the golden mean shows up in an average operation related to the power laws with exponential cut-off.

The invisible hand of information is the great shaper of the natural and social world. Continuous communication between self-constructing agents leads to an endless variation in their constitutive structures, involving both production and degradation processes which stem out from a generative blueprint or from any other form of a slowly changing 'identity'. The most common outcome, the preferred signature of the 'invisible hand' of information that collectively these agents compose -let us again state the hypothesis- is the partitional canon. 


\section{$7 \quad$ Reductionism and Information}

Claude Shannon was once asked by a student, "Do you believe that machines can be made to think?" He answered "You bet. I'm a machine and so are you." (supportively quoted by Gell-Mann, 2001, p. 43). Reductionists, which are legion in the natural sciences, miss the most interesting aspect of life. They are not directly wrong concerning their mechanistic approach, indeed a useful avenue of analytical research, but they betray the openness of science when they dogmatically leave out of focus any emerging aspects unrelated to the successive levels of particulate building blocks -for instance the inherent evanescence of biological structures and compounds at every level of organization, that harmoniously integrate the production and degradation aspects and the communication with the environment. Here, information (informational) has been considered as the special way of existence that stems out from such paradoxical 'evanescent permanence.'

The living being can only be considered a 'mechanism' concerning the teleology of its (evolutionary) design -but, obviously, that was not the meaning implied by Shannon's quotation. Of course, being a material entity, the constitution of the living has to obey the mechanical 'forcings' emanated from its interaction with the boundary conditions, following natural law (like any other piece of matter). But at the same time, through the exchange of signals, the living state may alter its own state (generatively producing a new constitution, or reconfiguring the present one) so that new boundary conditions will effectively apply to the interactions with its environment. In this sense, the living has internal knowledge and may literally choose the landscapes of natural law which are most favorable for the advancement of its own life cycle. Rather than resorting to those especial, and rather vacuous, terms coined during the 70's and 80's (autopoiesis, autogenesis, selfproduction, etc.) we can just say that the living existence is informational.

The particulate complex we have mentioned at the Introduction, so much entrenched within the reductionist way of thinking, actually prevails in most fields of science and actively blocks the development of genuine informational analyses. For instance, atoms (or whatever ultimate particle we consider) are central explanatory elements not only in physics, and chemistry, but also in biology (genes as explanatory particles of the living, e.g., Darwin, Fisher, and sociobiologists), also in neuroscience and behavior (for instance, conceptualizations such as Pavlovian learning, schemata theory, or the memes), and in the social, economic and ethical arenas (social atomism, individualism, and the doctrines of social utilitarism), and not to forget that atomism shines in linguistics and phonetic analysis too.

Looking for a fundamental explanation in any of these realms is tantamount as searching for its elementary building blocks, the "letters" whose combinations form the different levels of structure in the Galilean book of Nature. As Mcluhan $(1962,1964)$ and Needham (1962) have pointed out, the atomistic complex of thought was historically produced by cultures immersed in the alphabetic system of literacy. (Seemingly, archaic notions closer to the idea of "balance" and fecundity in nature were superseded by the new analytic mentality created by alphabets, perhaps in the shift from matriarchal styles of thinking to patriarchal ones; see for instance the interesting essay by Ortega y Gaset on 'Oknos, the rope-maker'). Philosopher Mary Midgley (2001) has poignantly criticized the atomistic thought which underlies reductionist and specialized visions of science, while William Abler (1989) has strongly emphasized the universal importance of the particulate forms of information as the only viable strategy for the construction of complex systems, either in the natural or in the artificial.

Of course, advancing the information science fields would imply far more than criticizing the blind spots of reductionism in natural science (comments from leading figures in yesteryear's 'heterodox' biological fields such as protein degradation, apoptosis, development, etc., are really revealing). Such partial blindness actually impinges into many other branches of our system of knowledge (particularly in those devoted to 'complex systems' where reductionism should be complemented necessarily by integrationism). 
We have socially built an enormous edifice of learning, treated almost as an homogeneous construction stemming out from the rules for the material but, quite probably, endowed in most of its disciplinary floors and corridors with the very paradoxical properties of the informational 'stuff' (power laws included). Almost any aspect of human societies, in spite of misguiding mechanistic terms such as market forces, social masses, political forces, and so on, belongs to the informational, per se, we may say.

In the economics proper, there are a number of organizational items such as money, prices, markets, firms, management, accounting practices ('balances'), stock markets, financial systems, futures, etc., that directly show the imprinting of the informational and bear a sophisticate story of social design behind them (Marijuán, 1998; Sánchez Vidal, 2000).

Besides, reflection on the most recent evolution of economic structures (telecommunications, entertainment, news, the arts, travel, tourism, fashion, shopping, sports, spectacles...) indicates an enormous influence by, say, social 'informational drives.' Indeed, a fast growing portion of the economy in the 'information society' is devoted to the massive production and channeling of just ephemeral novelty... Does this economico-cultural phenomenon of information mass-production relate to our most ancient past and even to our own nature as species -Homo informationalis? (Diamond, 1997; Hobart \& Schiffman, 1998). Unfortunately, contemporary neurosciences are far from producing a harmonious synthetic view on the nature of human 'information processing' and its underlying guidance upon social, cultural and economic evolution. Filling in this void may be one of the most interesting directions of advancement for the information science studies.

To conclude these 'informational' reflections, at the time being, there might be three paradigmatic directions for empirically establishing the foundations of information science. They would extend upon three really strategic scientific territories: cells, brains, and enterprises (societies). In the extent to which we gain conceptual command upon their common problems and problem-solving organizations, upon their differences and similarities, upon their communication and knowledge strategies -and we develop ad hoc new mathematical tools- a valuable synthesis would emerge. At the same time, other strategies theoretically inclined have to explore a variety of further directions. The bet here, just on a personal basis, is for empirically grounded reflections. Indeed, for the successful synthesis of a new information science, quite many approaches should cross-fertilize and add scientific value from very different perspectives.

\section{Summary}

A tentative new approach to the nature of information and a critique of the prevailing reductionist views have been presented. The new approach is mostly based on the molecular organization of the living cell. It is argued that the cellular balance between production and degradation of the constituent structures, performed in continuous communication with the environment and supported out from a genetic blueprint, may be the paradigm of further informational existences. Besides, the dynamic balance established by an informationally guided entity may produce a characteristic mathematical signature when re-enacted within competing populations of self-producing agents. The 'invisible hand' of information, biologically rooted, and extended both evolutionarily and socially through more, and more, complex agency realms, becomes the great shaper of our world. 


\section{References}

Abler, W.L. (1989) On the particulate principle of self-diversifying systems. Journal of Social and Biological Structures, 12, 1-13. Abraham, R., Jas, F. \& Russell, W. (1995) The Web Empowerment Book. Springer, New York.

Bak, P. (1996) How nature works: The science of self-organized criticality. Copernicus: Springer Verlag, New York.

Barham, J. (1996) A dynamical meaning of information. BioSystems, 38, 235-241.

Castells, M. (2000) The Rise of the Network Society. Blackwell Publ. Ltd., Oxford. 2nd edition.

Conrad, M. (1989) Physics and Biology: Towards a Unified Model. Applied Mathematics and Computation 32, 75-102.

Conrad, M. (1996) Cross-scale information processing in evolution, development and intelligence. BioSystems 38, 97-109.

Diamond, J. (1997) Guns, Germs and Steel: The Fates of Human Societies. W.W. Norton, New York.

Ebeling W. and Frömmel C., Entropy and predictability of information carriers. BioSystems 46 (1998) pp. 47-55.

Fleissner, P. \& Hofkirchner, W. (1998) The making of the information society. BioSystems, 46, 201-207.

Gasser S.M. (2002) Visualizing Chromatin Dynamics in Interphase Nuclei. Science 296 pp. 1412-1416.

Gell-Mann, M. (1994) The Quark and the Jaguar. Adventures in the Simple and the Complex. Little Brown, London.

Gell-Mann, M. (2001) Consciousness, Reduction, and Emergence: Some Remarks. Annals of the New York Academy of Sciences 929, 41-49.

Ho, M.V. (1995) Bioenergetics. The Open University, London.

Hobart, M.E. \& Schiffman, Z.S. (1998) Information Ages. The John Hopkins University Press, Baltimore

Hood, L. \& Galas, D. (2003) The digital code of DNA. Nature 421, 444-448.

Igamberviev, A.U. (1997) Quantum mechanical background of information processing in biological systems and its relation to symmetry-breaking. Symmetry: Culture and Science 8, 2, 193-205.

Javorszky, K. (1995) Zaragoza Lectures on Granularity Algebra, Mackinger-Verlag, Salzburg.

Javorszky, K. (2000) Interaction Between Sequences and Mixtures, J. of Theoretical Biology, 205, p. 663-665.

Javorszky, K. (2003) Information Processing in Auto-regulated Systems, Entropy, 5, 161-192.

Kay, L.E. (1993) The Molecular Vision of Life. Oxford University Press, New York.

Kirby K.G., Biological adaptabilities and quantum entropies. BioSystems 64 (2002) pp. 33-41.

Kitano H., Systems Biology: Towards System Level. In Foundations of Systems Biology, ed. by H. Kitano (The MIT Press,

Cambridge, 2001)

Lin S.K. (2001) The Nature of the Chemical Process. 1. Symmetry Evolution -Revised Information Theory, Similarity Principle and Ugly Symmetry. Int. J. Mol. Sci. 2 pp. 10-39.

Marijuán, P.C. (1994) Enzymes, automata and artificial cells. In Computing with biological metaphors, ed. by R.C. Paton. Chapman \& Hall, London, pp. 50-68.

Marijuán, P.C. (1996) Gloom in the society of enzymes. BioSystems, 38, 163-171

Marijuán, P.C. (1996b) Foundations of Information Science. FIS 1994 Conference Proceedings. Biosystems, 38, 87-96.

Marijuán, P.C. (1996c) Information and symmetry in the biological and social realm: New avenues of inquiry. Symmetry: Culture and Science 7, 3, 281-294.

Marijuán, P.C. (1998) Information and the unfolding of social life: molecular-biological resonances reaching up to the economy.

Biosystems 46: 145-151.

Marijuán, P.C. (2002) Bioinformation: untangling the networks of life. BioSystems, 64, 11-118.

Marijuán, P.C. (2003) From Inanimate Molecules to Living Cells: The Informational Scaffolding of Life. In: Energy and Information Transfer in Biological Systems. (Ed. Franco Musumeci.) World Scientific Press, Singapore. In press.

Marijuán, P.C., \& Villarroel, M. (1998) On Information Theory Stumbling Blocks. Cybernetics and Human Knowing, 5, 4, 19-29.

Marijuán, P.C., Pastor, J. \& Villarroel, M. (1998) The Language of Cells: A Partitional Approach to Cellular Communication.

Symmetry: Culture and Science 9, 2-4, 383-392.

McLuhan, M. (1962) The Gutenberg Galaxy. University of Toronto Press, Toronto.

McLuhan, M. (1964) Understanding Media: The Extensions of Man. University of Toronto Press, Toronto.

Midgley, M. (2001) Science and Poetry. Routledge, London.

Needham, J. (1962) Science and Civilization in China. Cambridge University Press, Cambridge.

Overbeek, R. (1999) WIT citation in NetWatch (wit.mcs.anl.gov/WIT2). Science 285, 1323.

Peirce, C.S. (1931-58): Collected Papers vol. I-VIII. (eds.) Hartshorne and Weiss. Cambridge MA: Harvard University Press. CDROM version from Past Masters.

Rosen, R. (1991) Life itself. Columbia University Press, New York. 
Sánchez Vidal, A. (2000) La máquina del tiempo. In: El Bodegón. Edited by Fundación Museo del Prado. Galaxia Gutenberg, Madrid.

Scarrott, G. (1998) The Formulation of a Science of Information: An Engineering Perspective on the Natural Properties of Information. Cybernetics and Human Knowing, 5, 4, 7-17.

Stonier, T. (1990) Information and the Internal Structure of the Universe. Springer, London.

Villarroel, M. (2002) Information processing in a partitional framework. FIS 2002 electronic conference. http://www.mdpi.net/fis2002/browse.htm

Wolfram, S. (2002) A New Kind of Science. Wolfram Media Inc.

Zuboff, S. (1995) The Emperor's New Workplace. Scientific American, September 1995, 162-164.

Zurek, W.H. (1990) Complexity, Entropy, and the Physics of Information, New York: Addison-Wesley. 